\title{
An improved upper bound for the order of mixed graphs
}

\author{
C. Dalfó \\ Dept. de Matemàtiques, Universitat Politècnica de Catalunya, Barcelona, Catalonia \\ cristina.dalfo@upc.edu \\ M. A. Fiol \\ Dept. de Matemàtiques, Barcelona Graduate School of Mathematics, \\ Universitat Politècnica de Catalunya, Barcelona, Catalonia \\ miguel.angel.fiol@upc.edu \\ N. López \\ Dept. de Matemàtica, Universitat de Lleida, Lleida, Spain \\ nlopez@matematica.udl.es
}

July 19, 2018

\begin{abstract}
A mixed graph $G$ can contain both (undirected) edges and arcs (directed edges). Here we derive an improved Moore-like bound for the maximum number of vertices of a mixed graph with diameter at least three. Moreover, a complete enumeration of all optimal $(1,1)$-regular mixed graphs with diameter three is presented, so proving that, in general, the proposed bound cannot be improved.
\end{abstract}

Keywords: Mixed graph, Moore bound, network design, degree/diameter problem.

2010 MSC: 05C30, 05C35

\section{Introduction}

A mixed (or partially directed) graph $G=(V, E, A)$ consists of a set $V$ of vertices, a set $E$ of edges, or unordered pairs of vertices, and a set $A$ of arcs, or ordered pairs of vertices. Thus, $G$ can also be seen as a digraph having digons, or pairs



The research of C. Dalfó has also received funding from the European Union's Horizon 2020 research and innovation programme under the Marie Skłodowska-Curie grant agreement No 734922. 
of opposite arcs between some pairs of vertices. If there is an edge between vertices $u, v \in V$, we denote it by $u \sim v$, whereas if there is an arc from $u$ to $v$, we write $u \rightarrow v$. We denote by $r(u)$ the undirected degree of $u$, or the number of edges incident to $u$. Moreover, the out-degree [respectively, in-degree] of $u$, denoted by $z^{+}(u)$ [respectively, $z^{-}(u)$ ], is the number of arcs emanating from [respectively, to] $u$. If $z^{+}(u)=z^{-}(u)=z$ and $r(u)=r$, for all $u \in V$, then $G$ is said to be totally regular of degrees $(r, z)$, with $r+z=d$ (or simply $(r, z)$ regular). The length of a shortest path from $u$ to $v$ is the distance from $u$ to $v$, and it is denoted by $\operatorname{dist}(u, v)$. Note that $\operatorname{dist}(u, v)$ may be different from $\operatorname{dist}(v, u)$ when the shortest paths between $u$ and $v$ involve arcs. The maximum distance between any pair of vertices is the diameter $k$ of $G$. Given $i \leq k$, the set of vertices at distance $i$ from vertex $u$ is denoted by $G_{i}(u)$.

As in the case of (undirected) graphs and digraphs, the degree/diameter problem for mixed graphs calls for finding the largest possible number of vertices $N(r, z, k)$ in a mixed graph with maximum undirected degree $r$, maximum directed outdegree $z$, and diameter $k$. A bound for $N(r, z, k)$ is called a Moore(like) bound. It is obtained by counting the number of vertices of a Moore tree $M T(u)$ rooted at a given vertex $u$, with depth equal to the diameter $k$, and assuming that for any vertex $v$ there exists a unique shortest path of length at most $k$ (with the usual meaning when we see $G$ as a digraph) from $u$ to $v$. The number of vertices in $M T(u)$, which is denoted by $M(r, z, k)$, was given by Buset, Amiri, Erskine, Miller, and Pérez-Rosés [2], and it is the following:

$$
M(r, z, k)=A \frac{u_{1}^{k+1}-1}{u_{1}-1}+B \frac{u_{2}^{k+1}-1}{u_{2}-1},
$$

where

$$
\begin{aligned}
v & =(z+r)^{2}+2(z-r)+1 \\
u_{1} & =\frac{z+r-1-\sqrt{v}}{2}, \quad u_{2}=\frac{z+r-1+\sqrt{v}}{2}, \\
A & =\frac{\sqrt{v}-(z+r+1)}{2 \sqrt{v}}, \quad B=\frac{\sqrt{v}+(z+r+1)}{2 \sqrt{v}} .
\end{aligned}
$$

This bound applies when $G$ is totally regular with degrees $(r, z)$. Moreover, if we bound the total degree $d=r+z$, the largest number is always obtained when $r=0$ and $z=d$. That is, when the mixed graph has no (undirected) edges. In Table 1 we show the values of (1) when $r=d-z$, with $0 \leq z \leq d$, for different values of $d$ and diameter $k$. In particular, when $z=0$, the bound corresponds to the Moore bound for graphs (numbers in bold).

\section{A new upper bound}

An alternative approach for computing the bound given by (1) is the following (see also [4]). Let $G$ be a $(r, z)$-regular mixed graph with $d=r+z$. Given 


\begin{tabular}{||l|ccccc||}
\hline \hline$d \backslash k$ & 1 & 2 & 3 & 4 & 5 \\
\hline 1 & $\mathbf{2}$ & $z+\mathbf{2}$ & $2 z+\mathbf{2}$ & $z^{2}+2 z+\mathbf{2}$ & $2 z^{2}+2 z+\mathbf{2}$ \\
2 & $\mathbf{3}$ & $z+\mathbf{5}$ & $4 z+\mathbf{7}$ & $z^{2}+9 z+\mathbf{9}$ & $5 z^{2}+16 z+\mathbf{1 1}$ \\
3 & $\mathbf{4}$ & $z+\mathbf{1 0}$ & $6 z+\mathbf{2 2}$ & $\boldsymbol{z}^{2}+22 z+\mathbf{4 6}$ & $8 z^{2}+66 z+\mathbf{9 4}$ \\
4 & $\mathbf{5}$ & $z+\mathbf{1 7}$ & $8 z+\mathbf{5 3}$ & $z^{2}+41 z+\mathbf{1 6 1}$ & $11 z^{2}+176 z+\mathbf{4 8 5}$ \\
5 & $\mathbf{6}$ & $z+\mathbf{2 6}$ & $10 z+\mathbf{1 0 6}$ & $z^{2}+66 z+\mathbf{4 2 6}$ & $14 z^{2}+370 z+\mathbf{1 7 0 6}$ \\
\hline \hline
\end{tabular}

Table 1: Moore bounds according to (1).

a vertex $v$ and for $i=0,1, \ldots, k$, let $N_{i}=R_{i}+Z_{i}$ be the maximum possible number of vertices at distance $i$ from $v$. Here, $R_{i}$ is the number of vertices that, in the corresponding tree rooted at $v$, are adjacent by an edge to their parents; and $Z_{i}$ is the number of vertices that are adjacent by an arc from their parents. Then,

$$
N_{i}=R_{i}+Z_{i}=R_{i-1}((r-1)+z)+Z_{i-1}(r+z) .
$$

That is,

$$
\begin{aligned}
R_{i} & =R_{i-1}(r-1)+Z_{i-1} r, \\
Z_{i} & =R_{i-1} z+Z_{i-1} z,
\end{aligned}
$$

or, in matrix form,

$$
\left(\begin{array}{c}
R_{i} \\
Z_{i}
\end{array}\right)=\left(\begin{array}{cc}
r-1 & r \\
z & z
\end{array}\right)\left(\begin{array}{c}
R_{i-1} \\
Z_{i-1}
\end{array}\right)=\cdots=M^{i}\left(\begin{array}{c}
R_{0} \\
Z_{0}
\end{array}\right)=M^{i}\left(\begin{array}{c}
0 \\
1
\end{array}\right),
$$

where $\boldsymbol{M}=\left(\begin{array}{cc}r-1 & r \\ z & z\end{array}\right)$ and, by convenience, $R_{0}=0$ and $Z_{0}=1$. Therefore,

$$
N_{i}=R_{i}+Z_{i}=\left(\begin{array}{ll}
1 & 1
\end{array}\right) M^{i}\left(\begin{array}{l}
0 \\
1
\end{array}\right) .
$$

Consequently, after summing a geometric matrix progression, the order of $M T(u)$ turns out to be

$$
M(r, z, k)=\sum_{i=0}^{k} N_{i}=\frac{1}{r+2 z-2}\left(\begin{array}{ll}
1 & 1
\end{array}\right)\left(\boldsymbol{M}^{k+1}-\boldsymbol{I}\right)\left(\begin{array}{c}
r \\
z
\end{array}\right),
$$

with $r+2 z \neq 2$, that is, except for the cases $(r, z)=(0,1)$ and $(r, z)=(2,0)$, which correspond to a directed and undirected cycle, respectively.

Alternatively, note that $N_{i}$ satisfies an easy linear recurrence formula (see again Buset, El Amiri, Erskine, Miller, and Pérez-Rosés [2]). Indeed, from (2) and (4) we have that $Z_{i}=z\left(N_{i-1}-Z_{i-1}\right)+z Z_{i-1}=z N_{i-1}$ and, hence,

$$
\begin{aligned}
N_{i} & =(r+z) N_{i-1}-R_{i-1}=(r+z) N_{i-1}-\left(N_{i-1}-Z_{i-1}\right) \\
& =(r+z-1) N_{i-1}+z N_{i-2}, \quad i=2,3, \ldots
\end{aligned}
$$


with initial values $N_{0}=1$ and $N_{1}=r+z$.

In this context, Nguyen, Miller, and Gimbert [6] showed that the bound in (1) is not attained for diameter $k \geq 3$ and, hence, that mixed Moore graphs do not exist in general. More precisely, they proved that there exists a pair of vertices $u, v$ such that there are two different paths of length $\leq k$ from $u$ to $v$. When there exist exactly two such paths, the usual terminology is to say that $v$ is the repeat of $u$, and this is denoted by writing $\operatorname{rep}(u)=v$ (see, for instance, Miller and Širán [5]). Extending this concept, we denote by $\operatorname{Rep}(u)$ the set (or multiset) of vertices $v$ such that there are $\nu \geq 2$ paths of length $\leq k$ from $u$ to $v$, in such a way that each $v$ appears $\nu-1$ times in $\operatorname{Rep}(u)$. (In other words, we could say that vertex $v$ is "repeated" or "revisited" $\nu-1$ times when reached from $u$.) Then, as a consequence, the number $N$ of vertices of $G$ must satisfy the bound

$$
N \leq|M T(u)|-|\operatorname{Rep}(u)|=M(r, z, k)-|\operatorname{Rep}(u)| .
$$

We use this simple idea in the proof of our main result.

Theorem 2.1. The order $N$ of a $(r, z)$-regular mixed graph $G$ with diameter $k \geq 3$ satisfies the bound

$$
N \leq M(r, z, k)-r
$$

where $M(r, z, k)$ is given by (1).

Proof. It is clear that we can assume that there are no parallel arcs or edges. Let $u$ be a vertex with edges to the vertices $v_{1}, \ldots, v_{r}$ and arcs to the vertices $u_{1}, \ldots, u_{z}$. For each $i=1, \ldots, r$, let $v_{i 1}, \ldots, v_{i z}$ be the vertices adjacent (through arcs) from $v_{i}$. (The situation in the case $r=z=2$ is depicted in Figure 1 , where the dashed lines represent paths.) Now, for some fixed $i=1, \ldots, r$ and $j=1, \ldots, z$, let us consider the following possible cases for the distance from a vertex in $\left\{u_{1}, \ldots, u_{z}\right\}$ to vertex $v_{i j}$ :

(i) If, for some $h=1, \ldots, z$, we have $\operatorname{dist}\left(u_{h}, v_{i j}\right)<k$, then there exist two paths of length at most $k$ from $u$ to $v_{i j}$ and, hence, $v_{i j} \in \operatorname{Rep}(u)$ (note that this includes the case $u_{h}=v_{i j}$ ).

(ii) If, for some $h=1, \ldots, z$, we have $\operatorname{dist}\left(u_{h}, v_{i j}\right)=k$ and the shortest path from $u_{h}$ to $v_{i j}$ goes through $v_{i}$, then there are two paths of length $\leq k$ from $u$ to $v_{i}$ (one of length 1 and the other of length $k$ ). Hence, $v_{i} \in \operatorname{Rep}(u)$. In fact, notice that, in this case, $\operatorname{dist}\left(u_{h}, v_{i \ell}\right)=k$ for every $\ell=1, \ldots, z$.

If, for every $h=1, \ldots, z$, we have $\operatorname{dist}\left(u_{h}, v_{i j}\right)=k$, let $w_{i j l}$ denote, for $\ell=$ $1, \ldots, z$, the predecessor vertices to $v_{i j}$ in the paths (of length $k$ ) from every $u_{h}$ to $v_{i j}$ (see the dashed lines in Figure 1). Now we have again two cases:

(iii) If, for some $\ell, \ell^{\prime}=1, \ldots, z$, we have $w_{i j \ell}=w_{i j \ell^{\prime}}$, then there are two paths of length $k$ from $u$ to $w_{i j \ell}$. Thus, $w_{i j \ell} \in \operatorname{Rep}(u)$. 


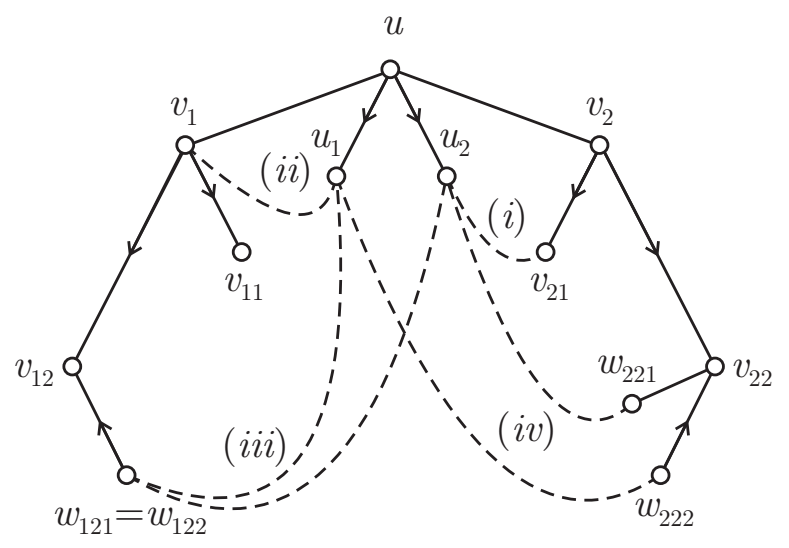

Figure 1: Repeated vertices in a (2,2)-regular mixed graph: $(i) v_{21} \in \operatorname{Rep}(u)$; (ii) $v_{1} \in \operatorname{Rep}(u)$; (iii) $w_{121} \in \operatorname{Rep}(u) ;($ iv $) w_{221} \in \operatorname{Rep}(u)$.

(iv) Otherwise, since $z^{-}\left(v_{i j}\right)=z$, there must be at least one $\ell$ such that $w_{i j \ell} v_{i j}$ is an edge. But, in this case, there are two paths from $u$ to $w_{i j \ell}$ of length at most $k(\geq 3)$ and, so, $w_{i j \ell} \in \operatorname{Rep}(u)$.

As a consequence, we see that, for each $i=1, \ldots, r$ there is a vertex, which is either $v_{i}, v_{i j}$, or $w_{i j \ell}$, belonging to $\operatorname{Rep}(u)$. Moreover, different values of $i$ lead to different repeated vertices, so that the paths from $u$ to them must be also different. In any case, the multiset $\operatorname{Rep}(u)$ has at least $r$ elements, and the result follows.

The new upper bound $M(r, z, k)-r$ for diameter $k \geq 3$ can be even improved for certain cases, as the next proposition states.

Proposition 2.2. Let $G$ be a $(r, z)$-regular mixed graph of diameter $k \geq 3$ with $\operatorname{order} N$. If $r$ and $z$ are odd, and $k \equiv 2 \bmod 3$, then

$$
N \leq M(r, z, k)-r-1 .
$$

Proof. The proof is based on a parity argument. Namely, since $r$ is odd, $N$ must be even. Thus, let us check the parity of $M(r, z, k)-r=\sum_{i=0}^{k} N_{i}-r$. Let $\pi_{i} \in\{0,1\}$ denote the parity of $N_{i}$ in the obvious way. If $z$ is odd, we have that $\pi_{0}=1, \pi_{1}=0$ and, from (6) we get the recurrence $\pi_{i}=\pi_{i-1}+\pi_{i-2}$ for $i \geq 2$. This gives the following sequence for the $\pi_{i}{ }^{\prime}$ s: $1,0,1,1,0,1,1,0,1,1, \ldots$ Thus, $\sum_{i=0}^{k} N_{i}$ is even for every $k \equiv 2 \bmod 3$. Then, as $r$ is odd, we get the result. 


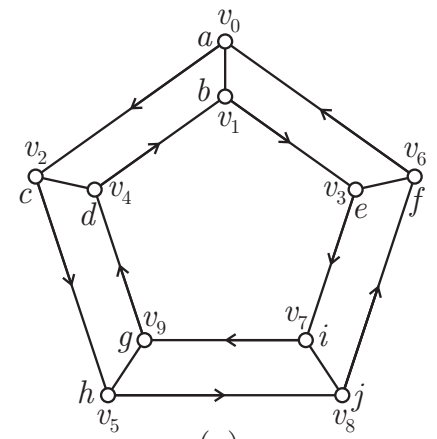

(a)

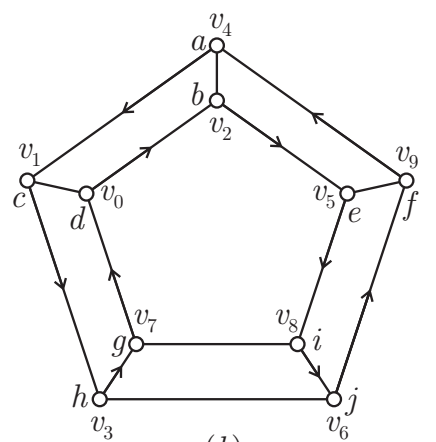

$(b)$

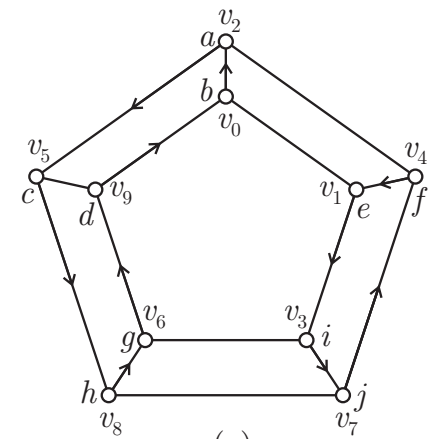

(c)

Figure 2: The unique three non-isomorphic $(1,1)$-regular mixed graphs with diameter $k=3$ and order $N=10$.

\section{The case of $(1,1)$-regular mixed graphs with diameter three}

In this section we show that the upper bound (7) is attained for exactly three mixed graphs in the case $r=z=1$ and $k=3$.

Proposition 3.1. Let $G$ be a $(1,1)$-regular mixed graph with diameter $k=3$ and maximum order $N=10$ given by (7). Then, $G$ is isomorphic to one of the three mixed graphs depicted in Figure 2.

Proof. We divide the proof according to the four cases $(i)-(i v)$ given in Theorem 2.1. Let $u$ be any vertex of $G$. The remaining vertices of $G$ fall into one of the sets $G_{i}(u)$, according to their corresponding distance $i \in\{1,2,3\}$ from $u$. Then, $\left|G_{1}(u)\right|=2$, and it is easy to see that $\left|G_{2}(u)\right|=3$ and $\left|G_{3}(u)\right|=4$ since, otherwise, $G$ would have order $N<M(1,1,3)-1=10$. Now, observe that case (i) is impossible since $\operatorname{dist}\left(u_{1}, v_{11}\right)<3$ would imply $\left|G_{3}(u)\right|<4$. Also, case (iii) is not possible simply because $z=1$. So, let us suppose that we are in case (ii), that is, $\operatorname{dist}\left(u_{1}, v_{11}\right)=3$ and the shortest path from $u_{1}$ to $v_{11}$ goes through $v_{1}$. Hence, $G$ contains one of the two induced mixed subgraphs depicted in Figure 3 (from now on, we follow the vertex labeling in this figure, where $v_{0}=u, v_{2}=u_{1}$ and $v_{3}=v_{11}$ ). Next, we proceed in detail with case (iia) and we leave to the reader cases $(i i b)$ and $(i v)$, where similar reasoning leads to the same mixed graphs.

Due to its regularity, $G$ must contain the edge $v_{7} \sim v_{8}$. Moreover, every vertex of $G$ is at distance $\leq 3$ from $v_{2}$ except $v_{6}$. This means that there must exist an arc $x \rightarrow v_{6}$, where $x \in\left\{v_{8}, v_{9}\right\}$.

- Let $x=v_{8}$. Another arc $y \rightarrow v_{9}$ is needed to have $\operatorname{dist}\left(v_{1}, v_{9}\right) \leq 3$, where $y \in\left\{v_{6}, v_{7}\right\}$.

- If $y=v_{6} \rightarrow v_{9}$ we have just two possibilities to complete the regularity of the mixed graph: 


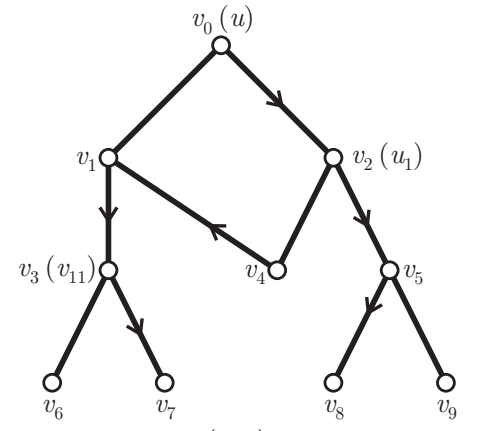

(iia)

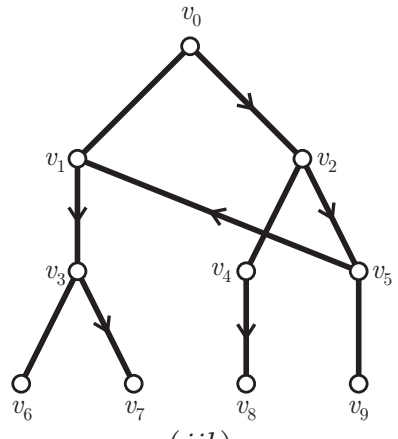

(iib)

Figure 3: The two cases derived from (ii) according to Theorem 2.1 when $r=1, z=1$ and $k=3$.



(iia1)

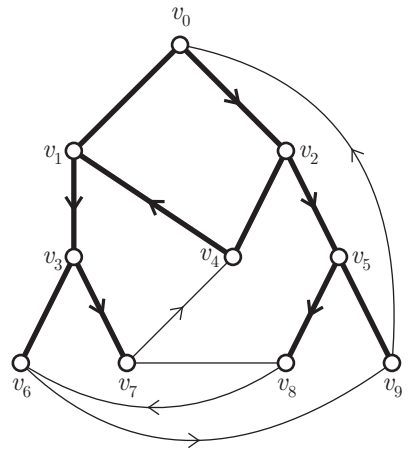

(iia2)

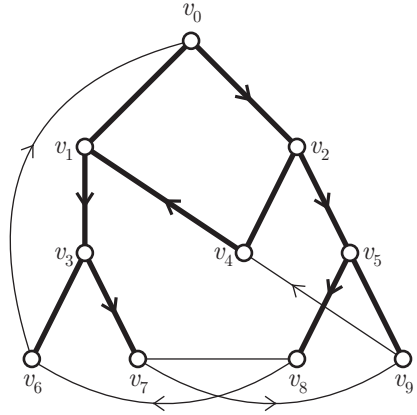

(iia3)

Figure 4: Three cases derived from (iia) giving non-isomorphic mixed graphs.

- The remaining arcs are $v_{7} \rightarrow v_{0}$ and $v_{9} \rightarrow v_{4}$, which yield the mixed graph of Figure 4(iia1), which is isomorphic to the one in Figure 2(b).

- The last arcs are $v_{7} \rightarrow v_{4}$ and $v_{9} \rightarrow v_{0}$, in which case we obtain the mixed graph of Figure 4(iia2), which is isomorphic to the one in Figure 2(c).

- If $y=v_{7} \rightarrow v_{9}$, we have again two possibilities:

- The arcs $v_{6} \rightarrow v_{0}$ and $v_{9} \rightarrow v_{4}$ yield the mixed graph of Figure 4(iia3), which is isomorphic to the one in Figure 2(a).

- The arcs $v_{6} \rightarrow v_{4}$ and $v_{9} \rightarrow v_{0}$ give rise to a mixed graph isomorphic to the one in Figure 2(b). 
A scheme of the above cases is the following.

$$
x=v_{8} \rightarrow v_{6} \Rightarrow\left\{\begin{array}{l}
y=v_{6} \rightarrow v_{9} \Rightarrow\left\{\begin{array}{l}
v_{7} \rightarrow v_{0} \& v_{9} \rightarrow v_{4} \rightsquigarrow(b) \\
\text { or } \\
v_{7} \rightarrow v_{4} \& v_{9} \rightarrow v_{0} \rightsquigarrow(c)
\end{array}\right. \\
\text { or } \begin{array}{l}
y=v_{7} \rightarrow v_{9}
\end{array} \Rightarrow\left\{\begin{array}{l}
v_{6} \rightarrow v_{0} \& v_{9} \rightarrow v_{4} \rightsquigarrow(a) \\
\text { or } \\
v_{6} \rightarrow v_{4} \& v_{9} \rightarrow v_{0} \rightsquigarrow(b)
\end{array}\right.
\end{array}\right.
$$

- Let $x=v_{9}$. We must add the arc $v_{7} \rightarrow v_{9}$ in order to have $\operatorname{dist}\left(v_{1}, v_{9}\right) \leq 3$. Now, to complete the mixed graph we have two possibilities:

- The $\operatorname{arcs} v_{6} \rightarrow v_{0}$ and $v_{8} \rightarrow v_{4}$ yield a mixed graph isomorphic to the one in Figure 2(b).

- The arcs $v_{6} \rightarrow v_{4}$ and $v_{8} \rightarrow v_{0}$ complete a mixed graph isomorphic to the one in Figure 2(c).

Schematically,

$$
x=v_{9} \rightarrow v_{6} \Rightarrow v_{7} \rightarrow v_{9} \Rightarrow\left\{\begin{array}{l}
v_{6} \rightarrow v_{0} \& v_{8} \rightarrow v_{4} \rightsquigarrow(b) \\
\text { or } \\
v_{6} \rightarrow v_{4} \& v_{8} \rightarrow v_{0} \rightsquigarrow(c)
\end{array}\right.
$$

This completes the proof.

Note that the mixed graph in Figure 2(a) is the line digraph of the cycle $C_{5}$ (seen as a digraph, so that each edge corresponds to a digon). It is also the Cayley graph of the dihedral group $D_{5}=\left\langle r, s \mid r^{5}=s^{2}=(r s)^{2}=1\right\rangle$, with generators $r$ and $s$. The spectrum of this mixed graph is that of the $C_{5}$ cycle plus a 0 with multiplicity 5 . Namely,

$$
\operatorname{sp} G=\left\{2,\left(-\frac{1}{2}+\frac{\sqrt{5}}{2}\right)^{2}, 0^{5},\left(-\frac{1}{2}-\frac{\sqrt{5}}{2}\right)^{2}\right\} .
$$

This is because $G$ is the line digraph of $C_{5}$. As a consequence, the only difference between $\operatorname{sp} G$ and $\operatorname{sp} C_{5}$ are the additional 0's (see Balbuena, Ferrero, Marcote, and Pelayo [1].) In fact, the mixed graphs of Figures 2(b) and 2(c) are cospectral with $G$, and can be obtained by applying a recent method to obtain cospectral digraphs with a locally line digraph. The right modifications to obtain the mixed graphs $(b)$ and $(c)$ from mixed graph $(a)$ are depicted in Figure 5. For more details, see Dalfó and Fiol [3].

Two other interesting characteristics of these mixed graphs are the following:

- Each of the three mixed graphs is isomorphic to its converse (where the directions of the arcs are reversed). 


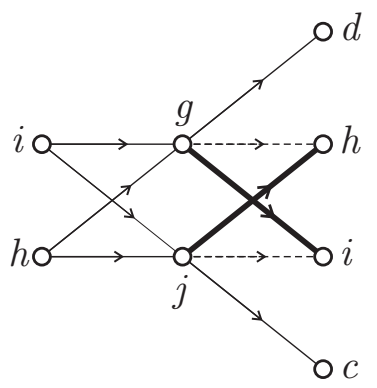

$(a) \rightarrow(b)$



$(b) \rightarrow(c)$

Figure 5: The method for obtaining the cospectral digraphs of Figure 2.

- Each of these mixed graphs can be obtained as a proper orientation of the so-called Yutsis graph of the $15 j$ symbol of the second kind (see Yutsis, Levinson, and Vanagas [7]). This is also called the pentagonal prim graph. Notice that it has girth 4 and, curiously, its diameter is 3 , in every of its considered orientations here.

The result of Proposition 3.1 could prompt us to look for a whole family of $(1,1)$-regular mixed graphs attaining the upper bound $M(1,1, k)-1$ for any diameter $k \geq 3$. Nevertheless, as a consequence of Proposition 2.2, this is not possible, since such a bound cannot be attained for some values of $k$.

Corollary 3.2. Let $G$ be a $(1,1)$-regular mixed graph with $N$ vertices and diameter $k=2+3 s$ with $s \geq 1$. Then,

$$
N \leq \theta_{1} \phi_{1}^{k+1}+\theta_{2} \phi_{2}^{k+1}-4,
$$

where $\theta_{1,2}=1 \pm \frac{2}{\sqrt{5}}$ and $\phi_{1,2}=\frac{1}{2}(1 \pm \sqrt{5})$.

Proof. Apply Proposition 2.2 with $r=z=1$ and $M(1,1, k)$ computed from (1).

Note that, in this last case, (6) yields the recurrence $N_{i}=N_{i-1}+N_{i-2}$, with $N_{0}=1$ and $N_{1}$, so defining a Fibonacci sequence. In fact, with the usual numbering of such a sequence $\left(F_{1}=1, F_{2}=1, F_{3}=2, \ldots\right)$, we have $M(1,1, k)=F_{k+4}-2$ and so, for the case under consideration, (9) becomes

$$
N \leq F_{k+4}-4 .
$$

Acknowledgments. The authors would like to thank an anonymous referee whose useful comments lead to a significant improvement of the manuscript. This research was partially supported by AGAUR under project 2017SGR1087 (C. D. and M. A. F.). The author N. L. has been supported, in part, by grant MTM2013-46949-P from the Ministerio de Economía y Competitividad, and 2014SGR1666 from the Catalan Research Council. 


\section{References}

[1] C. Balbuena, D. Ferrero, X. Marcote, and I. Pelayo, Algebraic properties of a digraph and its line digraph, J. Interconnection Networks 04 (2003), no. $4,377-393$.

[2] D. Buset, M. El Amiri, G. Erskine, M. Miller, and H. Pérez-Rosés, A revised Moore bound for partially directed graphs, Discrete Math. 339 (2016), no. 8, 2066-2069.

[3] C. Dalfó and M.A. Fiol, Cospectral digraphs from locally line digraphs, Linear Algebra Appl. 500 (2016) 52-62.

[4] C. Dalfó, M. A. Fiol, and N. López, Sequence mixed graphs, Discrete Appl. Math. 219 (2017) 110-116.

[5] M. Miller and J. Širáň, Moore graphs and beyond: A survey of the degree/diameter problem, Electron. J. Combin. 20(2) (2013) \#DS14v2.

[6] M. H. Nguyen, M. Miller, and J. Gimbert, On mixed Moore graphs, Discrete Math. 307 (2007) 964-970.

[7] A. P. Yutsis, L. B. Levinson, and V. V. Vanagas, Mathematical Apparatus of the Theory of Angular Momentum, Israel Program for Sci. Transl. Ltd., Jerusalem, 1962. 